\title{
Quasi-ISS/ISDS Reduced-Order Observers and Quantized Output Feedback for Interconnected Systems
}

\author{
Sergey Dashkovskiy and Lars Naujok
}

\begin{abstract}
In this paper the notion of quasi-input-to-state dynamical stability (quasi-ISDS) for reduced-order observer design is introduced. It combines the main advantage of ISDS over input-to-state stability (ISS), namely the memory fading effect, with reduced-order observers to obtain quantitative information about the state estimate error. As a second topic, interconnections of nonlinear systems are investigated and quasiISS reduced-order observers for the subsystems are designed when there exist suitable error Lyapunov functions. As an application of this concept, we prove that quantized output feedback stabilization for each subsystem and the overall system is achievable, when the systems possess a quasi-ISS reducedorder observer and a state feedback law that yields ISS for each subsystem and therefor the overall system with respect to measurement errors.
\end{abstract}

\section{INTRODUCTION}

The notion of quasi-input-to-state stability (quasi-ISS) for observers was introduced in [19]. There, a quasi-ISS reduced-order observer for nonlinear dynamical systems was designed and quantized output feedback stabilization was investigated. The design of observers and the problem of output-feedback stabilization was investigated for example in [13], [14], [12].

In [7] and [6] the property input-to-state dynamical stability (ISDS) was investigated, which is equivalent to the ISS property and has some advantages over ISS. One of these advantages is the so-called memory fading effect. Fading memory estimates were first studied in [17] and further studied for example in [9].

It is known for ISS (ISDS) systems that the influence of the "older" signals on the current state is essentially smaller then the influence of the recent ones. However the ISS estimation of trajectories does not take this into account. The advantage of the ISDS estimation is that it takes this dissipative property into account. In particular, if the input tends to zero, then the ISDS estimate will tend to zero, whereas the ISS estimate depends on the supremum norm of the input.

In this paper the approaches of reduced-order observers and the ISDS property are combined, which results in the introduction of quasi-ISDS reduced-order observers, which have the advantage that the recent disturbance of the measurement is taken into account.

Studying the ISS property, introduced in [20], for largescale interconnected systems establishes a small-gain condition as in [2], [3], [8], [10]. The ISDS property for large-scale systems was studied in [1].

Sergey N. Dashkovskiy and Lars Naujok are with the Zentrum für Technomathematik, Universität Bremen, Postfach 330440, 28334 Bremen, Germany, $\{$ dsn, larsnaujok $\}$ @math.uni-bremen. de
We design quasi-ISS reduced-order observers for subsystems of large-scale interconnected systems, from which an observer for the whole system can be designed. Furthermore we investigate the quantized output feedback stabilization for large-scale systems, which is based on the works [15], [19].

The paper is organized as follows: Section II contains some basic notions. The quasi-ISDS property is introduced in Section III. Section IV includes the design of quasi-ISS reduced-order observers for large-scale interconnected systems. The quantized output feedback for large-scale systems can be found in Section V. Finally, Section VI concludes the paper.

\section{PRELIMINARIES}

By $x^{T}$ we denote the transposition of a vector $x \in$ $\mathbb{R}^{n}, n \in \mathbb{N}$, furthermore $\mathbb{R}_{+}:=[0, \infty)$ and $\mathbb{R}_{+}^{n}$ denotes the positive orthant $\left\{x \in \mathbb{R}^{n}: x \geq 0\right\}$ where we use the standard partial order for $x, y \in \mathbb{R}^{n}$ given by

$x \geq y \Leftrightarrow x_{i} \geq y_{i}, i=1, \ldots, n$ and $x \geq y \Leftrightarrow \exists i: x_{i}<y_{i}$.

We denote the standard Euclidean norm in $\mathbb{R}^{n}$ by $|\cdot|$ and the supremum norm of a function $f$ by $\|f\|_{[a, b]}$, where $[a, b], a \leq b$ is an interval. $\nabla V$ denotes the gradient of a function $V$.

We consider general nonlinear systems of the form

$$
\dot{x}=f(x, u), y=h(x),
$$

where $x \in \mathbb{R}^{N}$ is the state, $u \in \mathbb{R}^{M}$ is a control input, $y \in \mathbb{R}^{P}$ is the output, function $f: \mathbb{R}^{N} \times \mathbb{R}^{M} \rightarrow \mathbb{R}^{N}$ is locally Lipschitz in $x$ uniformly in $u$ and function $h: \mathbb{R}^{N} \rightarrow \mathbb{R}^{P}$ is continuously differentiable with locally Lipschitz derivative (called a $C_{L}^{1}$ function). In addition, it is assumed $f(0,0)=0$ and $h(0)=0$.

A state observer for the system (1) is of the form

$$
\dot{\hat{\xi}}=F(\bar{y}, \hat{\xi}, u), \hat{x}=H(\bar{y}, \hat{\xi}, u),
$$

where $\hat{\xi} \in \mathbb{R}^{L}$ is the observer state, $\hat{x} \in \mathbb{R}^{N}$ is the estimate of the system state $x$ and $\bar{y} \in \mathbb{R}^{P}$ is the measurement of $y$ that may be disturbed by $d: \bar{y}=y+d$. We denote the state estimation error by

$$
\tilde{x}=\hat{x}-x .
$$

For the next sections we need: 
Definition 2.1: We define the following classes of functions:

$$
\begin{aligned}
\mathcal{K}:=\left\{\gamma: \mathbb{R}_{+} \rightarrow \mathbb{R}_{+} \mid\right. & \gamma \text { is continuous, } \gamma(0)=0 \\
& \text { and strictly increasing }\}
\end{aligned}
$$

$\mathcal{K}_{\infty}:=\{\gamma \in \mathcal{K} \mid \gamma$ is unbounded $\}$

$\mathcal{L}:=\left\{\gamma: \mathbb{R}_{+} \rightarrow \mathbb{R}_{+} \mid \gamma\right.$ is continuous and strictly decreasing with $\left.\lim _{t \rightarrow \infty} \gamma(t)=0\right\}$

$\mathcal{K} \mathcal{L}:=\left\{\beta: \mathbb{R}_{+} \times \mathbb{R}_{+} \rightarrow \mathbb{R}_{+} \mid \beta\right.$ is continuous,

$$
\beta(\cdot, t) \in \mathcal{K}, \beta(r, \cdot) \in \mathcal{L}, \forall t, r \geq 0\}
$$

$\mathcal{K} \mathcal{L} \mathcal{D}:=\{\mu \in \mathcal{K} \mathcal{L} \mid \mu(r, t+s)=\mu(\mu(r, t), s), \forall r, t, s \geq 0\}$

\section{QUASI-ISDS REDUCED-ORDER OBSERVERS}

In this section we introduce the quasi-ISDS property and give a motivating example for the introduction. Then we show that the reduced-order observer designed in Theorem 1 in [19] has the quasi-ISDS property provided that an error ISDS Lyapunov function exists.

We start with the following definition:

Definition 3.1: System (2) is called a quasi-ISDS observer for the system (1) if there exist functions $\tilde{\mu} \in \mathcal{K} \mathcal{L} \mathcal{D}, \tilde{\eta} \in \mathcal{K}_{\infty}$ and for each $K>0$ a function $\tilde{\gamma}_{K}^{\text {ISDS }} \in \mathcal{K}_{\infty}$ such that

$$
|\tilde{x}(t)| \leq \max \left\{\tilde{\mu}\left(\tilde{\eta}\left(\left|\tilde{x}_{0}\right|\right), t\right), \underset{\tau \in[0, t]}{\operatorname{ess} \sup _{\tilde{\mu}}} \tilde{\mu}\left(\tilde{\gamma}_{K}^{\mathrm{ISDS}}(|d(\tau)|), t-\tau\right)\right\},
$$

whenever $\|u\|_{[0, t]} \leq K$ and $\|x\|_{[0, t]} \leq K$.

The notion of ISDS was introduced in [7]. Here and in [1] the advantages of ISDS over ISS were discussed. One of these advantages is the memory-fading effect. For quasiISDS observer this means that the recent disturbance of the measurement is taken into account, whereas the quasiISS observer takes into account the supremum norm of the disturbance (see the Definition 1 in [19]).

The motivation of the introduction of quasi-ISDS observers will be illustrated by the following example.

Example 3.2: Consider the system in the Example 1 in [19]

$$
\dot{x}=-x+x^{2} u, y=x,
$$

where $\dot{\hat{x}}=-\hat{x}+y^{2} u$ is an observer. We consider the perturbed measurement $\bar{y}=y+d$, with $d=e^{-t \frac{1}{10}}$. Then the error dynamics becomes

$$
\dot{\tilde{x}}=-\tilde{x}+2 x u d+u d^{2} .
$$

This system is ISS and ISDS from $d$ to $\tilde{x}$ when $u(t)$ and $x(t)$ are bounded. Let $u \equiv 1$ be constant, then the estimations of the error dynamics are displayed in Figure 1 for $x_{0}=\tilde{x}_{0}=$ 0.2 . Here the ISS estimation takes the maximal value of $d$ into account, whereas the ISDS estimation has the so-called memory-fading effect.

We assume that there exists a global coordinate change $z=\phi(x)$ such that the system (1) is globally diffeomorphic to a system with linear output of the form

$$
\dot{z}=\left[\begin{array}{c}
\dot{z}_{1} \\
\dot{z}_{2}
\end{array}\right]=\left[\begin{array}{c}
\tilde{f}_{1}\left(z_{1}, z_{2}, u\right) \\
\tilde{f}_{2}\left(z_{1}, z_{2}, u\right)
\end{array}\right]=f(z, u), y=z_{1},
$$

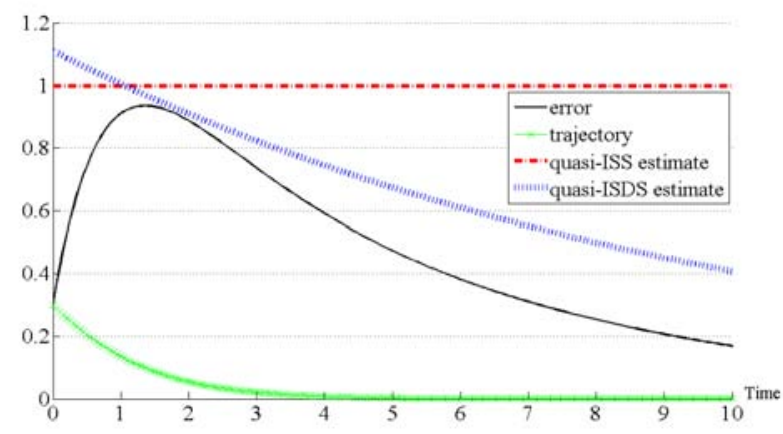

Fig. 1. Displaying of the trajectory, error, quasi-ISS and quasi-ISDS estimate of system (3).

where $z_{1} \in \mathbb{R}^{P}$ and $z_{2} \in \mathbb{R}^{N-P}$.

For the results in this paper we need the following assumption, where we use reduced-order error Lyapunov functions. Error Lyapunov functions were first introduced in [16] and in [11] the equivalence of the existence of an error Lyapunov function and the existence of an observer was shown.

Assumption 3.3: Let $\varepsilon>0$ be given. There exist a $C_{L}^{1}$ function $l: \mathbb{R}^{P} \rightarrow \mathbb{R}^{N-P}$, a $C^{1}$ function $V: \mathbb{R}^{N-P} \rightarrow \mathbb{R}$, functions $\alpha, \tilde{\eta} \in \mathcal{K}_{\infty}$ and $\tilde{\mu} \in \mathcal{K} \mathcal{L} \mathcal{D}$ such that

$$
\begin{aligned}
& \frac{|e|}{1+\varepsilon} \leq V(e) \leq \tilde{\eta}(|e|),\left|\frac{\partial V}{\partial e}(e)\right| \leq \alpha(|e|) \\
& \frac{\partial V}{\partial e}(e)\left(\left[\tilde{f}_{2}\left(z_{1}, e+z_{2}, u\right)+\frac{\partial l}{\partial z_{1}}\left(z_{1}\right) \tilde{f}_{1}\left(z_{1}, e+z_{2}, u\right)\right]\right. \\
&\left.-\left[\tilde{f}_{2}\left(z_{1}, z_{2}, u\right)+\frac{\partial l}{\partial z_{1}}\left(z_{1}\right) \tilde{f}_{1}\left(z_{1}, z_{2}, u\right)\right]\right) \\
& \leq-(1+\varepsilon) g(|e|)
\end{aligned}
$$

where $\tilde{\mu}$ solves the equation

$$
\frac{\mathrm{d}}{\mathrm{d} t} \tilde{\mu}(r, t)=-g(\tilde{\mu}(r, t)), \quad r, t>0
$$

for a locally Lipschitz continuous function $g: \mathbb{R}_{+} \rightarrow \mathbb{R}_{+}$ and there exist a function $\tilde{\alpha} \in \mathcal{K}_{\infty}$ such that

$$
\tilde{\alpha}(s) \alpha(s) \leq g(s), s \in \mathbb{R}_{+} .
$$

The next lemma is a counterpart of Theorem 1 in [19], where a quasi-ISS reduced-order observer for the system (4) was designed. Here the design is the same, but it provides a quasiISDS reduced-order observer for system (4).

Lemma 3.4: Under Assumption 3.3 the system

$$
\begin{aligned}
& \dot{\hat{\xi}}=\tilde{f}_{2}(\bar{y}, \hat{\xi}-l(\bar{y}), u)+\frac{\partial l}{\partial z_{1}}(\bar{y}) \tilde{f}_{1}(\bar{y}, \hat{\xi}-l(\bar{y}), u), \\
& \hat{z}_{1}=\bar{y}, \quad \hat{z}_{2}=\hat{\xi}-l(\bar{y}),
\end{aligned}
$$

where $\hat{\xi} \in \mathbb{R}^{N-P}$ is the observer state and $\hat{z}_{1}, \hat{z}_{2}$ are the estimates of $z_{1}$ and $z_{2}$, respectively, becomes a quasi-ISDS reduced-order observer for the system (4).

The proof goes along the lines of the proof of Theorem 1 in [19] with corresponding changes according to Definition 3.1 and Assumption 3.3. 


\section{LARGE-SCALE INTERCONNECTED SYSTEMS}

In this section we consider large-scale interconnected systems. We design quasi-ISS reduced-order observers for each subsystem and therefore the whole system, provided that the small-gain condition is satisfied.

We consider $n \in \mathbb{N}$ interconnected systems of the form

$$
\dot{x}_{i}=f_{i}\left(y_{1}, \ldots, x_{i}, \ldots, y_{n}, u_{i}\right), y_{i}=h_{i}\left(x_{i}\right),
$$

$i=1, \ldots, n$, where $x_{i} \in \mathbb{R}^{N_{i}}$ is the state of the $i$ th subsystem, $u_{i} \in \mathbb{R}^{M_{i}}$ are control inputs, $y_{i} \in \mathbb{R}^{P_{i}}$ are the outputs, functions $f_{i}: \mathbb{R}^{\sum_{j \neq i} P_{j}+N_{i}+M_{i}} \rightarrow \mathbb{R}^{N_{i}}$ are locally Lipschitz in $\left(y_{1}^{T}, \ldots, x_{i}^{T}, \ldots, y_{n}^{T}\right)^{T}$ uniformly in $u_{i}$ and functions $h_{i}: \mathbb{R}^{N_{i}} \rightarrow \mathbb{R}^{P_{i}}$ are continuously differentiable with locally Lipschitz derivatives. In addition, it is assumed $f_{i}(0, \ldots, 0)=0$ and $h_{i}(0)=0$.

The state observer of the $i$ th subsystem is of the form

$$
\begin{aligned}
\dot{\hat{\xi}}_{i} & =F_{i}\left(\bar{y}_{1}, \ldots, \bar{y}_{n}, \hat{\xi}_{i}, u_{i}\right), \\
\hat{x}_{i} & =H_{i}\left(\bar{y}_{1}, \ldots, \bar{y}_{n}, \hat{\xi}_{i}, u_{i}\right),
\end{aligned}
$$

$i=1, \ldots, n$, where $\hat{\xi}_{i} \in \mathbb{R}^{L_{i}}$ is the observer state of the $i$ th subsystem, $\hat{x}_{i} \in \mathbb{R}^{N_{i}}$ is the estimate of the system state $x_{i}$ and $\bar{y}_{i} \in \mathbb{R}^{P_{i}}$ is the measurement of $y_{i}$ that may be disturbed by $d_{i}: \bar{y}_{i}=y_{i}+d_{i}$. We note the state estimation error of the $i$ th subsystem by $\tilde{x}_{i}:=\hat{x}_{i}-x_{i}$. Next, we define the quasi-ISS property extending Definition 1 in [19] for interconnected systems.

Definition 4.1: The $i$ th subsystem of (8) is called a quasiISS observer for the $i$ th subsystem of (7) if there exist a function $\tilde{\beta}_{i} \in\{\mathcal{K} \mathcal{L}\}$ and for each $K_{i}>0$ functions $\tilde{\gamma}_{i}^{K_{i}}, \tilde{\gamma}_{i j}^{K_{i}} \in\{\mathcal{K}\}_{\infty}, j=1, \ldots, n, j \neq i$ such that

$$
\begin{aligned}
& \left|\tilde{x}_{i}(t)\right| \\
\leq & \left.\max \left\{\tilde{\beta}_{i}\left(\left|\tilde{x}_{i}^{0}\right|\right), t\right), \max _{j \neq i} \tilde{\gamma}_{i j}^{K_{i}}\left(\left\|d_{j}\right\|_{[0, t]}\right), \tilde{\gamma}_{i}^{K_{i}}\left(\left\|d_{i}\right\|_{[0, t]}\right)\right\},
\end{aligned}
$$

whenever $\left\|u_{i}\right\|_{[0, t]} \leq K_{i}$ and $\left\|x_{j}\right\|_{[0, t]} \leq K_{i}, j=1, \ldots, n$. We assume that there exists a global coordinate change $z_{i}=$ $\phi_{i}\left(x_{i}\right)$ with $x_{i}=\left(y_{1}^{T}, \ldots, x_{i}^{T}, \ldots, y_{n}^{T}\right)^{T}$ such that the $i$ th subsystem of (7) is globally diffeomorphic to a system with linear output of the form

$$
\begin{aligned}
\dot{z}_{i} & =\left[\begin{array}{c}
\dot{z}_{1 i} \\
\dot{z}_{2 i}
\end{array}\right]=\left[\begin{array}{l}
f_{1 i}\left(y_{1}, \ldots, z_{1 i}, z_{2 i}, \ldots, y_{n}, u_{i}\right) \\
f_{2 i}\left(y_{1}, \ldots, z_{1 i}, z_{2 i}, \ldots, y_{n}, u_{i}\right)
\end{array}\right] \\
& =f_{i}\left(y_{1}, \ldots, z_{i}, \ldots, y_{n}, u_{i}\right), \\
y_{i} & =z_{1 i}, \quad i=1, \ldots, n,
\end{aligned}
$$

where $z_{1 i} \in \mathbb{R}^{P_{i}}$ and $z_{2 i} \in \mathbb{R}^{N_{i}-P_{i}}, i=1, \ldots, n$.

Assumption 4.2: For each $i=1, \ldots, n$ there exist $C_{L}^{1}$ functions $l_{i}: \mathbb{R}^{P_{i}} \rightarrow \mathbb{R}^{N_{i}-P_{i}}$, a $C^{1}$ function $V_{i}: \mathbb{R}^{N_{i}-P_{i}} \stackrel{C_{L}}{\rightarrow}$
$\mathbb{R}$, functions $\alpha_{1 i}, \alpha_{2 i}, \alpha_{3 i}, \alpha_{4 i}, \in \mathcal{K}_{\infty}$ such that

$$
\begin{aligned}
\alpha_{1 i}\left(\left|e_{i}\right|\right) & \leq V_{i}\left(e_{i}\right) \leq \alpha_{2 i}\left(\left|e_{i}\right|\right),\left|\frac{\partial V_{i}}{\partial e_{i}}\left(e_{i}\right)\right| \leq \alpha_{4 i}\left(\left|e_{i}\right|\right), \\
\frac{\partial V_{i}}{\partial e_{i}}\left(e_{i}\right) & \left(\left[f_{2 i}\left(y_{1}, \ldots, z_{1 i}, e_{i}+z_{2 i}, \ldots, y_{n}, u_{i}\right)\right.\right. \\
& \left.+\frac{\partial l_{i}}{\partial z_{1 i}}\left(z_{1 i}\right) f_{1 i}\left(y_{1}, \ldots, z_{1 i}, e_{i}+z_{2 i}, \ldots, y_{n}, u_{i}\right)\right] \\
& -\left[f_{2 i}\left(y_{1}, \ldots, z_{1 i}, z_{2 i}, \ldots, y_{n}, u_{i}\right)\right. \\
& \left.\left.+\frac{\partial l_{i}}{\partial z_{1 i}}\left(z_{1 i}\right) f_{1 i}\left(y_{1}, \ldots, z_{1 i}, z_{2 i}, \ldots, y_{n}, u_{i}\right)\right]\right) \\
& \leq-\alpha_{3 i}\left(\left|e_{i}\right|\right)
\end{aligned}
$$

and there exist class- $\mathcal{K}_{\infty}$ functions $\alpha_{i}$ such that

$$
\alpha_{i}(s) \alpha_{4 i} \leq \alpha_{3 i}(s) .
$$

The next theorem is a counterpart of the Theorem 1 in [19] for the design of an observer for each subsystem of an interconnected system.

Theorem 4.3: Under Assumption 4.2, the system

$$
\begin{aligned}
\hat{\dot{\xi}}_{i}= & f_{2 i}\left(\bar{y}_{1}, \ldots, \bar{y}_{i}, \hat{\xi}_{i}-l_{i}\left(\bar{y}_{i}\right), \ldots, \bar{y}_{n}, u_{i}\right) \\
& +\frac{\partial l_{i}}{\partial z_{1 i}}\left(\bar{y}_{i}\right) f_{1 i}\left(\bar{y}_{1}, \ldots, \bar{y}_{i}, \hat{\xi}_{i}-l_{i}\left(\bar{y}_{i}\right), \ldots, \bar{y}_{n}, u_{i}\right) \\
\hat{z}_{1 i}= & \bar{y}_{i}, \hat{z}_{2 i}=\hat{\xi}_{i}-l_{i}\left(\bar{y}_{i}\right)
\end{aligned}
$$

becomes a quasi-ISS reduced-order observer for the $i$ th subsystem of (9).

Proof: We define $\xi_{i}:=z_{2 i}+l_{i}\left(z_{1 i}\right)$. Then

$$
\begin{aligned}
\dot{z}_{1 i} & =f_{1 i}\left(y_{1}, \ldots, z_{1 i}, \xi_{i}-l_{i}\left(z_{1 i}\right), \ldots, y_{n}, u_{i}\right), \\
\dot{\xi}_{i} & =f_{2 i}\left(y_{1}, \ldots, z_{1 i}, \xi_{i}-l_{i}\left(z_{1 i}\right), \ldots, y_{n}, u_{i}\right) \\
& =: F_{i}\left(y_{1}, \ldots, z_{1 i}, \xi_{i}, \ldots, y_{n}, u_{i}\right), \\
y_{i} & =z_{1 i}, i=1, \ldots, n .
\end{aligned}
$$

The reduced-order observer (10) is written as $\hat{\xi}_{i}=$ $F_{i}\left(\bar{y}_{1}, \ldots, \bar{y}_{i}, \hat{\xi}_{i}, \ldots, \bar{y}_{n}, u_{i}\right)$. Let $e:=\hat{\xi}_{i}-\xi_{i}$. We use the shorthand for $j=1,2$

$\hat{f}_{j i}^{d}=$

$f_{j i}\left(y_{1}+d_{1}, \ldots, z_{1 i}+d_{i}, \hat{\xi}_{i}-l_{i}\left(z_{1 i}+d_{i}\right), \ldots, y_{n}+d_{n}, u_{i}\right)$, $f_{j i}^{d}=$

$f_{j i}\left(y_{1}+d_{1}, \ldots, z_{1 i}+d_{i}, \xi_{i}-l_{i}\left(z_{1 i}+d_{i}\right), \ldots, y_{n}+d_{n}, u_{i}\right)$,

$f_{j i}=f_{j i}\left(y_{1}, \ldots, z_{1 i}, \xi_{i}-l_{i}\left(z_{1 i}\right), \ldots, y_{n}, u_{i}\right)$,

then we have

$$
\begin{aligned}
& \dot{V}_{i}\left(e_{i}\right) \\
= & \frac{\partial V_{i}}{\partial e_{i}}\left(e_{i}\right)\left(\left[\hat{f}_{2 i}^{d}+\frac{\partial l_{i}}{\partial z_{1 i}}\left(z_{1 i}+d_{i}\right) \hat{f}_{1 i}^{d}\right]\right. \\
& \left.-\left[f_{2 i}+\frac{\partial l_{i}}{\partial z_{1 i}}\left(z_{1 i}\right) f_{1 i}\right]\right)
\end{aligned}
$$




$$
\begin{aligned}
& \leq-\alpha_{3 i}\left(\left|e_{i}\right|\right)+\frac{\partial V_{i}}{\partial e_{i}}\left(e_{i}\right)\left(F_{i}\left(\bar{y}_{1}, \ldots, \bar{y}_{i}, \xi_{i}, \ldots, \bar{y}_{n}, u_{i}\right)\right. \\
&\left.-F_{i}\left(y_{1}, \ldots, y_{i}, \xi_{i}, \ldots, y_{n}, u_{i}\right)\right) \\
& \leq- \alpha_{3 i}\left(\left|e_{i}\right|\right)+\max \left\{\gamma_{i}\left(z_{11}, \ldots, y_{i}, \xi_{i}, \ldots, z_{1 n}, u_{i}\right) \rho_{i}\left(\left|d_{i}\right|\right),\right. \\
&\left.\max _{j \neq i} \gamma_{i j}\left(z_{11}, \ldots, y_{i}, \xi_{i}, \ldots, z_{1 n}, u_{i}\right) \rho_{i j}\left(\left|d_{j}\right|\right)\right\},
\end{aligned}
$$

where $\gamma_{i}, \gamma_{i j}$ are continuous positive functions and $\rho_{i}, \rho_{i j}$ are class- $\mathcal{K}$ functions such that

$$
\begin{aligned}
& \left|F_{i}\left(\bar{y}_{1}, \ldots, \bar{y}_{i}, \xi_{i}, \ldots, \bar{y}_{n}, u_{i}\right)-F_{i}\left(y_{1}, \ldots, y_{i}, \xi_{i}, \ldots, y_{n}, u_{i}\right)\right| \\
& \leq \max \left\{\gamma_{i}\left(z_{11}, \ldots, y_{i}, \xi_{i}, \ldots, z_{1 n}, u_{i}\right) \rho_{i}\left(\left|d_{i}\right|\right)\right. \\
& \left.\max _{j \neq i} \gamma_{i j}\left(z_{11}, \ldots, y_{i}, \xi_{i}, \ldots, z_{1 n}, u_{i}\right) \rho_{i j}\left(\left|d_{j}\right|\right)\right\}
\end{aligned}
$$

whose existence can be shown using similar results in [4], [5]. It follows that for an arbitrary $\varepsilon_{i} \in(0,1)$, we have

$$
\begin{aligned}
& \left|e_{i}\right| \geq \\
& \alpha_{i}^{-1}\left(( 1 - \varepsilon _ { i } ) \operatorname { m a x } \left\{\gamma_{i}\left(z_{11}, \ldots, y_{1}, \xi_{i}, \ldots, z_{1 n}, u_{i}\right) \rho_{i}\left(\left|d_{i}\right|\right),\right.\right. \\
& \left.\left.\max _{j \neq i} \gamma_{i j}\left(z_{11}, \ldots, y_{i}, \xi_{i}, \ldots, z_{1 n}, u_{i}\right) \rho_{i j}\left(\left|d_{j}\right|\right)\right\}\right) \\
& \Rightarrow \dot{V}_{i} \leq-\varepsilon_{i} \alpha_{3 i}\left(\left|e_{i}\right|\right) .
\end{aligned}
$$

Under the conditions that $\left|z_{j}(\tau)\right| \leq K_{i}$ and $\left|u_{i}(\tau)\right| \leq$ $K_{i}, j=1, \ldots, n$ for $0 \leq \tau \leq t$ it can be shown that there exist a function $\bar{\beta}_{i} \in \mathcal{K} \mathcal{L}$, functions $\bar{\gamma}_{i}^{K_{i}}, \bar{\gamma}_{i j}^{K_{i}} \in\{\mathcal{K}\}_{\infty}, j=$ $1, \ldots, n, j \neq i$ such that

$$
\begin{aligned}
& \left|e_{i}(t)\right| \leq \\
& \left.\max \left\{\bar{\beta}_{i}\left(\left|e_{i}^{0}\right|\right), t\right), \max _{j \neq i} \bar{\gamma}_{i j}^{K_{i}}\left(\left\|d_{j}\right\|_{[0, t]}\right), \bar{\gamma}_{i}^{K_{i}}\left(\left\|d_{i}\right\|_{[0, t]}\right)\right\} .
\end{aligned}
$$

Recalling (10), we have that

$$
\begin{aligned}
\tilde{z}_{i} & =\left[\begin{array}{c}
\tilde{z}_{1 i} \\
\tilde{z}_{2 i}
\end{array}\right]:=\left[\begin{array}{c}
\hat{z}_{1 i}-z_{1 i} \\
\hat{z}_{2 i}-z_{2 i}
\end{array}\right] \\
& =\left[\begin{array}{c}
d_{i} \\
e_{i}-l_{i}\left(z_{1 i}+d_{i}\right)+l_{i}\left(z_{1 i}\right)
\end{array}\right],
\end{aligned}
$$

which leads to

$$
\left|\tilde{z}_{i}\right| \leq\left|d_{i}\right|+\left|e_{i}\right|+\theta_{K_{i}}\left(\left|d_{i}\right|\right) \text { and }\left|e_{i}\right| \leq\left|\tilde{z}_{2 i}\right|+\theta_{K_{i}}\left(\left|d_{i}\right|\right),
$$

where $\theta_{K_{i}}\left(\left|d_{i}\right|\right)$ is a class- $\mathcal{K}$ function, parametrized by $K_{i}$ such that $\left|l\left(z_{1 i}+d_{i}\right)-l\left(z_{1 i}\right)\right| \leq \theta_{K_{i}}\left(\left|d_{i}\right|\right)$ when $\left|z_{1 i}\right| \leq K_{i}$. Together with (11) we obtain

$$
\begin{aligned}
& \quad\left|\tilde{z}_{i}(t)\right| \\
& \left.\leq \max \left\{\bar{\beta}_{i}\left(\left|e_{i}^{0}\right|\right), t\right), \max _{j \neq i} \bar{\gamma}_{i j}^{K_{i}}\left(\left\|d_{j}\right\|_{[0, t]}\right), \bar{\gamma}_{i}^{K_{i}}\left(\left\|d_{i}\right\|_{[0, t]}\right)\right\} \\
& \quad+\theta_{K_{i}}\left(\left|d_{i}(t)\right|\right)+\left|d_{i}(t)\right| \\
& \leq \max \left\{3 \bar{\beta}_{i}\left(\left(\left|\tilde{z}_{2 i}^{0}\right|+\theta_{K_{i}}\left(\left|d_{i}^{0}\right|\right)\right), t\right),\right. \\
& \left.\quad 3 \max _{j \neq i} \bar{\gamma}_{i j}^{K_{i}}\left(\left\|d_{j}\right\|_{[0, t]}\right), 3 \chi_{i}^{K_{i}}\left(\left\|d_{i}\right\|_{[0, t]}\right)\right\},
\end{aligned}
$$

where $\chi_{i}^{K_{i}}(r):=\max \left\{\bar{\gamma}_{i}^{K_{i}}(r), \theta_{K_{i}}(r), r\right\}$. By $\alpha(a+b) \leq$ $\max \{\alpha(2 a), \alpha(2 b)\}$ for $\alpha \in \mathcal{K}$ we have that

$$
\begin{aligned}
& \left|\bar{\beta}_{i}\left(\left(\left|\tilde{z}_{2 i}^{0}\right|+\theta_{K_{i}}\left(\left|d_{i}^{0}\right|\right)\right), t\right)\right| \\
\leq & \max \left\{\bar{\beta}_{i}\left(2\left|\tilde{z}_{2 i}^{0}\right|, t\right), \bar{\beta}_{i}\left(2 \theta_{K_{i}}\left(\left|d_{i}^{0}\right|\right), t\right)\right\} \\
\leq & \max \left\{\bar{\beta}_{i}\left(2\left|\tilde{z}_{2 i}^{0}\right|, t\right), 2 \theta_{K_{i}}\left(\left.|| d_{i}\right|_{[0, t]}\right)\right\}
\end{aligned}
$$

and with $\check{\beta}_{i}(r, t):=\bar{\beta}_{i}(2 r, t), \bar{\theta}_{K_{i}}:=2 \theta_{K_{i}}$ we obtain

$$
\begin{aligned}
& \left|\tilde{z}_{i}(t)\right| \\
\leq & \max \left\{3 \check{\beta}_{i}\left(\left|\tilde{z}_{2 i}^{0}\right|, t\right), 3 \bar{\theta}_{K_{i}}\left(\left\|d_{i}\right\|_{[0, t]}\right), 3 \max _{j \neq i} \bar{\gamma}_{i j}^{K_{i}}\left(\left\|d_{j}\right\|_{[0, t]}\right),\right. \\
& \left.3 \chi_{i}^{K_{i}}\left(|| d_{i} \|_{[0, t]}\right)\right\} \\
= & : \max \left\{\tilde{\beta}_{i}\left(\left|\tilde{z}_{i}^{0}\right|, t\right), \max _{j \neq i} \tilde{\gamma}_{i j}^{K_{i}}\left(\left\|d_{j}\right\|_{[0, t]}\right), \tilde{\gamma}_{i}^{K_{i}}\left(\left\|d_{i}\right\|_{[0, t]}\right)\right\},
\end{aligned}
$$

which proves that the system (10) is a quasi-ISS reduced order observer for the $i$ th subsystem.

Remark 4.4: This approach is also possible for the quasiISDS property, with similar calculations. It may happen that the decay rate becomes conservative, because the estimations above are conservative.

Now, if we define $P=\sum P_{i}, N=\sum N_{i}, M=\sum M_{i}$ $z:=\left(z_{1}^{T}, \ldots, z_{n}^{T}\right)^{T} \in \mathbb{R}^{N}, z_{1}:=\left(z_{11}^{T}, \ldots, z_{1 n}^{T}\right)^{T} \in \mathbb{R}^{P}$, $z_{2}:=\left(z_{21}^{T}, \ldots, z_{2 n}^{T}\right)^{T} \in \mathbb{R}^{P-N}, u:=\left(u_{1}^{T}, \ldots, u_{n}^{T}\right)^{T} \in$ $\mathbb{R}^{M}, d=\left(d_{1}^{T}, \ldots, d_{n}^{T}\right)^{T}$ and $f:=\left(f_{1}^{T}, \ldots, f_{n}^{T}\right)^{T}, \tilde{f}_{1}:=$ $\left(f_{11}^{T}, \ldots, f_{1 n}^{T}\right)^{T}, \tilde{f}_{2}:=\left(f_{21}^{T}, \ldots, f_{2 n}^{T}\right)^{T}$, then the system (9) can be written as a system of the form (4).

We collect all gains $\tilde{\gamma}_{i j}^{K_{i}}$ in a gain-matrix $\widetilde{\Gamma}$, which defines a map $\widetilde{\Gamma}: \mathbb{R}_{+}^{n} \rightarrow \mathbb{R}_{+}^{n}$ by

$$
\widetilde{\Gamma}(s):=\left(\max _{j} \tilde{\gamma}_{1 j}^{K_{1}}\left(s_{j}\right), \ldots, \max _{j} \tilde{\gamma}_{n j}^{K_{n}}\left(s_{j}\right)\right)^{T},
$$

$\forall s \in \mathbb{R}_{+}^{n}$, with $\tilde{\gamma}_{i i}^{K_{i}} \equiv 0$.

We say that $\widetilde{\Gamma}$ satisfies the small-gain condition if

$$
\Gamma(s) \nsupseteq s, \forall s \in \mathbb{R}_{+}^{n} \backslash\{0\} .
$$

For more details about this condition see [2], [3]. Note that if $\widetilde{\Gamma}$ satisfies the small-gain condition (13), then there exists an $\Omega$-path $\sigma=\left(\sigma_{1}, \ldots, \sigma_{n}\right)^{T}$ with respect to $\widetilde{\Gamma}$ (see [3], Theorem 5.2 and [18]).

With these considerations a quasi-ISS reduced-order observer for the overall system can be designed.

Lemma 4.5: Consider a system of the form (9). Assume that Assumption 4.2 and Theorem 4.3 hold true for each $i=$ $1, \ldots, n$. If $\widetilde{\Gamma}$ satisfies the small-gain condition (13), then the reduced-order error Lyapunov function $V$ as in Assumption 1 in [19] is given by $V=\max _{i}\left\{\sigma_{i}^{-1}\left(V_{i}\right)\right\}$ and the quasi-ISS reduced-order observer for the overall system is given by

$$
\hat{\xi}=\left(\hat{\xi}_{1}^{T}, \ldots, \hat{\xi}_{n}^{T}\right)^{T},
$$

and

$$
\begin{aligned}
& \hat{\dot{\xi}}=f_{2}(\bar{y}, \hat{\xi}-l(\bar{y}), u)+\frac{\partial l}{\partial z_{1}}(\bar{y}) f_{1}(\bar{y}, \hat{\xi}, u) \\
& \hat{z}_{1}=\bar{y}, \quad \hat{z}_{2}=\hat{\xi}-l(\bar{y}) .
\end{aligned}
$$

Proof: We give only an outline of the proof:

For vector valued functions $z=\left(z_{1}^{T}, \ldots, z_{n}^{T}\right)^{T}: \mathbb{R}_{+} \rightarrow$ $\mathbb{R}^{\sum_{i=1}^{n} N_{i}}$ with $z_{i}: \mathbb{R}_{+} \rightarrow \mathbb{R}^{N_{i}}$ and times $0 \leq t_{1} \leq t_{2}, t \in$ $\mathbb{R}_{+}$we define

$$
\begin{aligned}
|\tilde{z}(t)| & :=\left(\left|\tilde{z}_{1}(t)\right|, \ldots,\left|\tilde{z}_{n}(t)\right|\right)^{T} \in \mathbb{R}_{+}^{n}, \\
\|\boldsymbol{\|} d\|_{\left[t_{1}, t_{2}\right]} & :=\left(\left\|d_{1}\right\|_{\left[t_{1}, t_{2}\right]}, \ldots,\left\|d_{n}\right\|_{\left[t_{1}, t_{2}\right]}\right)^{T} \in \mathbb{R}_{+}^{n}, .
\end{aligned}
$$


For $t \in \mathbb{R}_{+}$and $s \in \mathbb{R}_{+}^{n}$ we define

$$
\begin{aligned}
\tilde{\gamma}_{K}\left(\|d\|_{\left[t_{1}, t_{2}\right]}\right) & :=\left(\tilde{\gamma}_{1}^{K_{1}}\left(\left\|d_{1}\right\|_{\left[t_{1}, t_{2}\right]}\right), \ldots, \tilde{\gamma}_{n}^{K_{n}}\left(\left\|d_{n}\right\|_{\left[t_{1}, t_{2}\right]}\right)\right)^{T}, \\
\tilde{\beta}(s, t) & :=\left(\tilde{\beta}_{1}\left(s_{1}, t\right), \ldots, \tilde{\beta}_{n}\left(s_{n}, t\right)\right)^{T} \in \mathbb{R}_{+}^{n} .
\end{aligned}
$$

Using Assumption 4.2 and Theorem 4.3 for each subsystem we arrive at

$$
\mathbf{|} \tilde{z}(t) \mathbf{I} \leq \max \left\{\tilde{\beta}\left(\mathbf{|} \tilde{z}_{0} \mathbf{l}\right), \widetilde{\Gamma}\left(\boldsymbol{\|} d \boldsymbol{\|}_{[0, t]}\right), \tilde{\gamma}\left(\|d\|_{[0, t]}\right)\right\}
$$

where the maximum for vectors is taken componentwise. Since the small-gain condition is satisfied it follows with similar arguments as for the proof of Theorem 11 in [2] that $\hat{\xi}=\left(\hat{\xi}_{1}^{T}, \ldots, \hat{\xi}_{n}^{T}\right)^{T}$ is a quasi-ISS reduced-order observer for the overall system. Similar to the proof of Theorem 5.3 in [3] it follows, that the reduced-order error Lyapunov function $V$ for the overall system is given by $V=\max _{i}\left\{\sigma_{i}^{-1}\left(V_{i}\right)\right\}$.

\section{QUANTIZED OUTPUT FEEDBACK}

In this section we first consider single systems and combine the quantized output feedback stabilization with the ISDS property as in Chapter V in [19]. Then we consider large-scale systems and give a counterpart to Proposition 1 in [19] for such kind of systems.

\section{A. Single systems}

We consider again one single system in the form (4). By an output quantizer we mean a piecewise constant function $q$ : $\mathbb{R}^{P} \rightarrow \mathcal{Q}$, where $\mathcal{Q}$ is a finite subset of $\mathbb{R}^{P}$. The quantization error is denoted by

$$
d:=q(y)-y,
$$

and the quantizer's range $M>0$ by $|y| \leq M$, which implies the error bound $|d| \leq \Delta, \Delta>0$.

Now suppose that Assumption 3.3 holds and a quasi-ISDS observer has been designed as in Lemma 3.4. With $d$ as in (15) the observer acts on the quantized output measurements $\bar{y}=q(y)$. Furthermore suppose that a controller is given in the form $u=k(z)$. We can now define a quantized output feedback law by

$$
u=k(\hat{z})=k(z+\tilde{z}),
$$

where $\hat{z}$ is the state estimate generated by the observer and $\tilde{z}=\hat{z}-z$ is the state estimation error. We impose on the feedback law:

Assumption 5.1: The system

$$
\dot{z}=f(z, k(\hat{z}))=f(z, k(z+\tilde{z}))
$$

is ISDS with respect to $\tilde{z}$, i.e.,

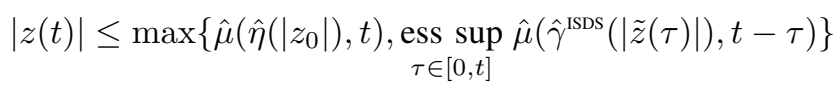

for some $\hat{\mu} \in \mathcal{K} \mathcal{L} \mathcal{D}, \hat{\eta}$ and $\hat{\gamma}^{\text {IsDs }} \in \mathcal{K}_{\infty}$.

For a detailed discussion for the case of an ISS controller we refer to [15].
The overall closed-loop system obtained by combining the plant, the observer and the control law can be written as

$$
\begin{aligned}
\dot{z}= & {\left[\begin{array}{c}
\dot{z}_{1} \\
\dot{z}_{2}
\end{array}\right]=\left[\begin{array}{c}
f_{1}\left(z_{1}, z_{2}, k(\hat{z})\right) \\
f_{2}\left(z_{1}, z_{2}, k(\hat{z})\right)
\end{array}\right], } \\
\dot{\hat{\xi}}= & f_{2}\left(q\left(z_{1}\right), \hat{\xi}-l\left(q\left(z_{1}\right)\right), k(\hat{z})\right) \\
& +\frac{\partial l}{\partial z_{1}}\left(q\left(z_{1}\right)\right) f_{1}\left(q\left(z_{1}\right), \hat{\xi}-l\left(q\left(z_{1}\right)\right), k(\hat{z})\right), \\
\hat{z}= & {\left[\begin{array}{c}
\hat{z}_{1} \\
\hat{z}_{2}
\end{array}\right]=\left[\begin{array}{c}
q\left(z_{1}\right) \\
\hat{\xi}-l\left(q\left(z_{1}\right)\right)
\end{array}\right] . }
\end{aligned}
$$

We know that for $e=\hat{\xi}-\xi$, where $\xi=z_{2}+l\left(z_{1}\right)$, the bound

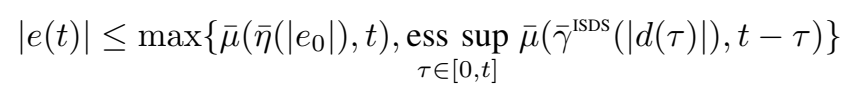

holds. Combining this with (16) and $|\tilde{z}| \leq|d|+|e|+\theta_{K}(|d|)$, we can show that

$$
\left|\left(\begin{array}{c}
z(t) \\
\hat{\xi}(t)
\end{array}\right)\right| \leq \max \left\{\mu\left(\eta\left(\left|\left(\begin{array}{c}
z_{0} \\
\hat{\xi}_{0}
\end{array}\right)\right|\right), t\right), \nu(d, t)\right\}
$$

is valid for $\|z\|_{[0, t]} \leq K$ and $\|u\|_{[0, t]}=\|k(\hat{z})\|_{[0, t]} \leq K$, where $\nu(d, t):=$ ess $\sup _{\tau \in[0, t]} \mu\left(\gamma^{\text {ISDS }}(|d(\tau)|), t-\tau\right)$.

\section{B. Large-scale systems}

Now, we consider again an interconnected system of the form 7 or 9 , respectively.

The output quantizer of the $i$ th subsystem is given by $q_{i}: \mathbb{R}^{P_{i}} \rightarrow \mathcal{Q}_{i}$, where $\mathcal{Q}_{i}$ is a finite subset of $\mathbb{R}^{P_{i}}$, the quantization error by $d_{i}:=q_{i}\left(y_{i}\right)-y_{i}$, the quantizer's range $M_{i}>0$ by $\left|y_{i}\right| \leq M_{i}$, which implies $\left|d_{i}\right| \leq \Delta_{i}$, where $\Delta_{i}>0$ is the error bound. We suppose that Assumption 4.2 holds and observer for the $i$ th subsystem has been designed by Theorem 4.3 , which acts on the quantized output measurements $\bar{y}_{i}=q_{i}\left(y_{i}\right)$.

Suppose that a controller of the $i$ th subsystem is given by $u_{i}=k_{i}\left(z_{i}\right)$ and the quantized output feedback law is defined by

$$
u_{i}:=k_{i}\left(\hat{z}_{i}\right)=k_{i}\left(z_{i}+\tilde{z}_{i}\right)
$$

where $\hat{z}_{i}$ is the state estimate generated by the observer and $\tilde{z}_{i}=\hat{z}_{i}-z_{i}$ is the state estimation error. In the rest of the paper we suppose the following:

Assumption 5.2: The $i$ th subsystem $\dot{z}_{i}=$ $f_{i}\left(y_{1}, \ldots, z_{i}, \ldots, y_{n}, k_{i}\left(\hat{z}_{i}\right)\right)$ is ISS with respect to $\tilde{z}_{i}$, i.e.,

$$
\begin{aligned}
& \left|z_{i}(t)\right| \\
\leq & \max \left\{\hat{\beta}_{i}\left(z_{i}^{0}, t\right), \max _{j \neq i} \hat{\gamma}_{i j}\left(\left\|\tilde{z}_{j}\right\|_{[0, t]}\right), \hat{\gamma}_{i}\left(\left\|\tilde{z}_{i}\right\|_{[0, t]}\right)\right\}
\end{aligned}
$$

for some $\hat{\beta}_{i} \in \mathcal{K} \mathcal{L}$ and $\hat{\gamma}_{i j}, \hat{\gamma}_{i} \in \mathcal{K}_{\infty}$.

We can show that for the $i$ th subsystem of the overall closed-loop system obtained by combining the plant, the observer and the control law it holds

$$
\begin{aligned}
& \left|\left(\begin{array}{c}
z_{i}(t) \\
\hat{\xi}_{i}(t)
\end{array}\right)\right| \\
\leq & \max \left\{\beta_{i}\left(\left|\begin{array}{c}
z_{i}^{0} \\
\hat{\xi}_{i}^{0}
\end{array}\right|, t\right), \max _{j \neq i} \gamma_{i j}^{K_{i}}\left(\left\|d_{j}\right\|_{[0, t]}\right), \gamma_{i}^{K_{i}}\left(\left\|d_{i}\right\|_{[0, t]}\right)\right\}
\end{aligned}
$$


for $\left\|z_{j}\right\|_{[0, t]} \leq K_{i}, \quad j=1, \ldots, n$ and $\left\|u_{i}\right\|_{[0, t]}=$ $\left\|k_{i}\left(\hat{z}_{i}\right)\right\|_{[0, t]} \leq K_{i}$.

Furthermore we can show, that if the small-gain condition (13) is satisfied for $\Gamma=\left(\gamma_{i j}\right)_{n \times n}$, which defines a map as in (12), then for the overall closed system it holds

$$
\left|\left(\begin{array}{c}
z(t) \\
\hat{\xi}(t)
\end{array}\right)\right| \leq \max \left\{\beta\left(\left|\begin{array}{c}
z^{0} \\
\hat{\xi}^{0}
\end{array}\right|, t\right), \gamma^{K}\left(\|d\|_{[0, t]}\right)\right\},
$$

where $z, \hat{\xi}, \beta$ and $\gamma^{K}$ are defined as in Lemma 4.5 and its proof.

Define $z^{1}=\left(z_{11}^{T}, \ldots, z_{1 n}^{T}\right)^{T}, l=\left(l_{1}^{T}, \ldots, l_{n}^{T}\right)^{T}$ and $\kappa_{l} \in$ $\mathcal{K}_{\infty}$ with $\left|l\left(z^{1}\right)\right| \leq \kappa_{l}\left(\left|z^{1}\right|\right), \forall z^{1}$ and $\kappa_{u} \in \mathcal{K}_{\infty}$ with $|k(z)| \leq$ $\kappa_{u}(|z|), \forall z$, so that we give a counterpart of Proposition 1 in [19] for interconnected systems.

Proposition 5.3:

1) Assume $\max \left\{\gamma_{i j}^{K_{i}}\left(\Delta_{j}\right), \gamma_{i}^{K_{i}}\left(\Delta_{i}\right)\right\} \leq M_{i}$ and

$$
\left|\left(\begin{array}{c}
z_{i}^{0} \\
\hat{\xi}_{i}^{0}
\end{array}\right)\right|<E_{i}^{0}
$$

where $E_{i}^{0}>0$ is such that $\beta_{i}\left(E_{i}^{0}, 0\right)=M_{i}$. Then the corresponding solution of the $i$ th subsystem of the overall closed-loop system satisfies

$$
\limsup _{t \rightarrow \infty}\left|\left(\begin{array}{c}
z_{i}(t) \\
\hat{\xi}_{i}(t)
\end{array}\right)\right| \leq \max \left\{\gamma_{i j}^{K_{i}}\left(\Delta_{j}\right), \gamma_{i}^{K_{i}}\left(\Delta_{i}\right)\right\} .
$$

2) Assume that 1) holds for $i=1, \ldots, n$. Define $M:=$ $\max M_{i}, \Delta:=\max \Delta_{i}, K:=\max \left\{M, \kappa_{u}(2 M+\Delta+\right.$ $\left.\left.\kappa_{l}(M+\Delta)\right)\right\}$ and suppose that $\Gamma$ satisfies the smallgain condition (13). Then the corresponding solution of the overall closed-loop system satisfies

$$
\limsup _{t \rightarrow \infty}\left|\left(\begin{array}{c}
z(t) \\
\hat{\xi}(t)
\end{array}\right)\right| \leq \gamma^{K}(\Delta) \text {. }
$$

The proof is omitted because of the limited space, but 1) goes along the line of the proof of Proposition 1 in [19] with corresponding changes to interconnected systems and 2) is an immediate consequence of 1) and the small-gain condition.

\section{CONCLUSIONS}

We introduced the quasi-ISDS property for observers, which main advantage over ISS is the memory fading effect. This was demonstrated in an example. We showed how to design quasi-ISS reduced-order observers for subsystems of interconnected systems. They were used to design an quasiISS reduced-order observer for the overall system under a small-gain condition.

As an application we showed that quantized output feedback stabilization for a subsystem is achievable, under the assumptions that the subsystem possesses a quasi-ISS reducedorder observer and a state feedback controller providing ISS with respect to measurement errors. If this holds for all subsystems of the large-scale system and the smallgain condition is satisfied, then quantized output feedback stabilization is also achievable for the overall system.

Future works are for example the investigation of the design of nonlinear output feedback control or nonlinear observers to satisfy the small-gain condition and the application of the results in this paper to the design of dynamic quantized interconnected control systems.

\section{ACKNOWLEDGMENTS}

Sergey Dashkovskiy and Lars Naujok are supported by the German Research Foundation (DFG) as part of the Collaborative Research Centre 637 "Autonomous Cooperating Logistic Processes: A Paradigm Shift and its Limitations".

\section{REFERENCES}

[1] Sergey Dashkovskiy and Lars Naujok. ISDS small-gain theorem and construction of ISDS Lyapunov functions for interconnected systems. Systems and Control Letters, 59(5):299-304, 2010.

[2] Sergey Dashkovskiy, Björn S. Rüffer, and Fabian R. Wirth. An ISS small gain theorem for general networks. Math. Control Signals Systems, 19(2):93-122, 2007.

[3] Sergey N. Dashkovskiy, Björn S. Rüffer, and Fabian R. Wirth. Small Gain Theorems for Large Scale Systems and Construction of ISS Lyapunov Functions. SIAM Journal on Control and Optimization, 48(6):4089-4118, 2010.

[4] R.A. Freeman and P.V. Kokotović. Global robustness of nonlinear systems to state measurement disturbances. In Proceedings of the 32nd IEEE CDC, pages 1507-1512, 1993.

[5] Randy A. Freeman and Petar V. Kokotović. Robust nonlinear control design. Systems \& Control: Foundations \& Applications. Birkhäuser Boston Inc., Boston, MA, 1996. State-space and Lyapunov techniques.

[6] Lars Grüne. Asymptotic behavior of dynamical and control systems under perturbation and discretization, volume 1783 of Lecture Notes in Mathematics. Springer-Verlag, Berlin, 2002.

[7] Lars Grüne. Input-to-state dynamical stability and its Lyapunov function characterization. IEEE Trans. Automat. Control, 47(9):14991504, 2002.

[8] Zhong-Ping Jiang and Yuan Wang. A generalization of the nonlinear small-gain theorem for large-scale complex systems. In Proceedings of the 7th WCICA., pages 1188-1193, June 2008.

[9] Iasson Karafyllis. A system-theoretic framework for a wide class of systems. II. Input-to-output stability. J. Math. Anal. Appl., 328(1):466486, 2007.

[10] Iasson Karafyllis and Zhong-Ping Jiang. A small-gain theorem for a wide class of feedback systems with control applications. SIAM J. Control Optim., 46(4):1483-1517 (electronic), 2007.

[11] Iasson Karafyllis and Costas Kravaris. Robust output feedback stabilization and nonlinear observer design. Systems Control Lett., 54(10):925-938, 2005.

[12] Iasson Karafyllis and Costas Kravaris. From continuous-time design to sampled-data design of observers. IEEE Trans. Automat. Control, 54(9):2169-2174, 2009.

[13] D. Karagiannis, A. Astolfi, and R. Ortega. Two results for adaptive output feedback stabilization of nonlinear systems. Automatica $J$. IFAC, 39(5):857-866, 2003.

[14] D. Karagiannis, Z. P. Jiang, R. Ortega, and A. Astolfi. Output-feedback stabilization of a class of uncertain non-minimum-phase nonlinear systems. Automatica J. IFAC, 41(9):1609-1615, 2005.

[15] D. Liberzon. Observer-based quantized output feedback control of nonlinear systems. In Proceedings of the 17th IFAC World Congress, 2008.

[16] L. Praly. On Observers with State Independent Error Lyapunov Function. In Proceedings of the 5th IFAC Symposium NOLCOS'01, pages 1425-1430, July 2001.

[17] Laurent Praly and Yuan Wang. Stabilization in spite of matched unmodeled dynamics and an equivalent definition of input-to-state stability. Math. Control Signals Systems, 9(1):1-33, 1996.

[18] B. S. Rüffer. Monotone inequalities, dynamical systems, and paths in the positive orthant of Euclidean $n$-space. Positivity., 14(2):257-283, 2010.

[19] Hyungbo Shim, Daniel Liberzon, and Jung-Su Kim. Quasi-ISS Reduced-Order Observers and Quantized Output Feedback. In Proceedings of the 48th IEEE CDC, pages 6680-6685, 2009.

[20] Eduardo D. Sontag. Smooth stabilization implies coprime factorization. IEEE Trans. Automat. Control, 34(4):435-443, 1989. 